\title{
THE STUDY AND DEVELOPMENT OF COMBINED ARTIFICIAL INTELLIGENCE ARCHITECTURES TO SOLVE IMPORTANT PROBLEMS IN COMPUTER VISION
}

\author{
Craig M. Arndt \\ Armstrong Laboratory \\ U.S. Air Force
}

\begin{abstract}
The practical usefulness of many important automated systems is currently dependent on the advancement of computer vision to a point where well defined engineering and computer science methods can be used in the development of practical equipment. In this proposal I will suggest an approach to developing practical robust computer vision systems. I propose to develop an intelligent architecture, in collaboration with research partners, which will permit the development of algorithms for implementation in specific engineering development projects as well as the expansion and validation of the theoretical understanding of computer and human vision and intelligence. In this paper I will describe a new approach to developing computer vision systems which will in. clude the development of an overall system based on three parts. First we will study aspects of human and computer vision and intelligence. The second part of the project will be the development of new AI algorithms for application to specific problems and issues in computer vision. The last part of the project will be the integration of these new algorithms and existing techniques under a new architecture so that more complex, less restricted applications can be addressed. These parts of the research and development effort should be worked at the same time and by the same team to maximize collaboration.
\end{abstract}

\section{Introduction}

The practical usefulness of many important automated systems is currently dependent on the advancement of computer vision to a point where well defined engineering and computer science methods can be used in the development of practical equipment. In this proposal I will suggest an approach to developing practical robust computer vision systems. I propose to develop an intelligent architecture, in collaboration with research partners, which will permit the development of algorithms for implementation in specific engineering development projects as well as the expansion and validation of the theoretical understanding of computer and human vision and intelligence.

\section{Problem}

There are a number of basic problems which limit the usability of computer vision systems as they are now designed. Current systems lack diversity, robustness, and flexibility in non-constrained environments. The largest problem is that there is a disconnect between the understanding of visual cognition and the reality of image processing

\section{Approach}

The approach to developing new computer vision systems will be to develop an overall system based on three important parts. First we will study aspects of human and computer vision and intelligence. The second part of the project will be the development of new AI algorithms for application to specific problems and issues in computer vision. The last part of the project will be the integration of these new algorithms and existing techniques under a new architecture so that more complex, less restricted applications can be addressed. These parts of the research and development effort should be worked at the same time and by the same team to maximize collaboration.

The first part of the project will include investigation of the architectures which make vision as we know it possible. Clearly vision is a function of intelligence as much as sensor input and image processing. In order for a vision system to have reasonable performance in a complex operating environment the architecture will need to collect, integrate, and direct multiple input sensors, and evaluate, maintain and 
update models of the current state of the operating environment. In the past most computer vision systems have concentrated on the processing of single sensor input image data. Human visual systems, on the other hand, use multiple sensor inputs,in addition to complex internal representation of the individual's environment in order to make decisions. New computer vision architectures will incorporate standard video input with active feedback and range sensor data to enhance the ability to collect important data. In order to deal with low data rates and extremely complex and varied tasks, humans use higher brain functions to enhances their vision system. By maintaining models of their environment, humans can perform complex tasks using many parts of their brain in parallel. Environmental modeling can also be used to enhance the performance of computer vision systems. In contrast to the traditional approaches of constructing and updating kalman filter-like representations, the new architecture will use the human model to evaluate the system state as a function of deviations from and movement with probabilistic models of task environments.

The second part of the project of the development/enhancement of computer vision is the development of new AI techniques for use in the vision system. Expert systems have been used in the past for encoding human expert knowledge in a problem solving domain. By using the rule-based structure of expert systems, we can develop systems which will monitor and enhance sensor integration, system state and environmental models and task processing. Predictive filtering results can then be evaluated with environmental models. The use of traditional and custom designed neural nets will enhance tracking and image possessing for landmark identification.

The last part of the project is the most challenging. It involves integrating and training new and traditional techniques into the new architecture. The ability to develop a system to function and perform multiple real world tasks will depend on the use of new AI techniques to implement the overall architectures. Expert Systems with the capability to learn and generalize in the manner of Neural Nets from various applications experiments will be developed to detect and update state changes. The Blackboard use of independent knowledge sources and opportunistic reasoning may be used to implement the overall architecture.

\section{Applications}

There are a number of important applications for bia sic and applied research in computer vision. Tht importance of computer vision to the ongoing devei. opment of robotics is key to many industries. Th." ability to "see" in a robust manner within a con plex unrestrained environment will allow robots 1 . perform many of the mosi important tasks we desir. them to do. Other applications of advances in computer vision include the areas of pattern recognition, including face recognition, and monitoring of scentes and subjects to name a few. The less direct but morc important application of computer vision research is the understanding of the complexities of human vision as it relates to human and computer intelligence as a whole. Understanding some of the key issues in how humans integrate intelligence and vision to perform complex tasks will allow us to design AI systems that can begin to perform tasks previously reserved for humans alone. In my position as a research engineer and project manager at the Armstrong lab, am currently doing research in several areas of computer vision and image processing. Some of the basic principles of combined artificial intelligence architecture discussed in this proposal have been implemented with considerable success in projects involving face recognition and landmark identification in 3-dimensional landscapes. The results of some of this research will be presented as an Invited paper at the SPIE Conference on Applications of Artificial Neurai Networks in April 1992.

\section{Architectures}

The goal of an expanded architecture is not only to improve performance of current systems and application but to allow computer vision to take on the aspects of intelligence which human vision systems exhibit. Humans use their vision system and other parts of their brain to gain what is termed Situational Awareness, that aspect of intelligence that allows humans to understand their environment and interact within it. Through the use of intelligent systems, we can incorporate a computer vision system for modeling the system state. By combining these systems, we have a system capable of system and environmental situational awareness. In order to realize the poten. tial for computer vision, more complex architectures are needed. I will examine four architectures that demonstrate how combined AI systems can be used to increase the performance of computer vision systems. These architectures are being used in my research and in that of a number of other researchers with greal 
PIPELINE ARCHITECTURE

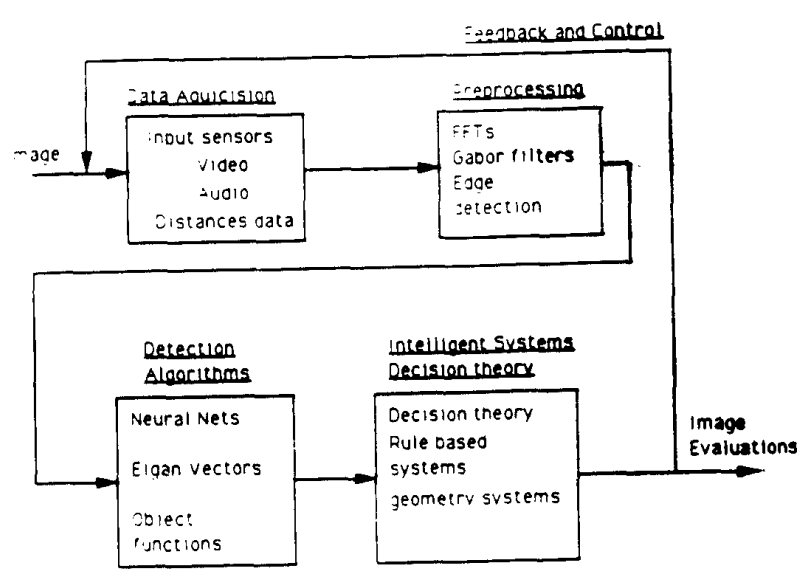

Figure 1:

success. The first approach is a pipeline architecture where the processing components are grouped based on their functions in the overall computer vision system, see figure 1 . The first component is the data acquisition. In this component, time sequencing of inputs and data fusion functions will be addressed. In the second, component image pre-processing will take place. These functions are similar to the transfer functions in the human vision system, which include edge detectors and filtering functions. The third component is used to look at the image and detect or identify parts of the image. The fourth component is the implementation of intelligent decisions about what the image is and its meaning. The last component of the system will close the loop for the system with feedback and control.

After the basic pipeline of the image processing is generated and calculated, it will then be possible to take second looks at the image. Human vision is capable of extracting additional data out of images using second looks and information about what to look for. Like humans, the computer vision system will examine the results of processed first look data and intelligently make decisions as to how to direct the data acquisition and preprocessing of second looks in order to maximize obtaining the most useful information from the image. The computer vision system
NETS and LISP ARCHITECTURE

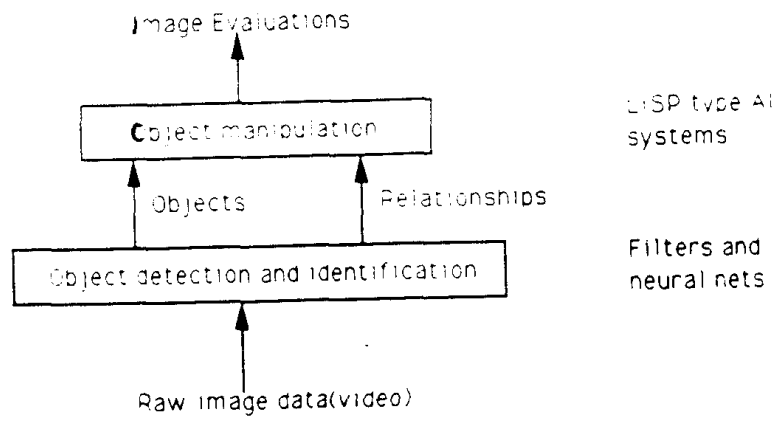

Figure 2:

will, therefore, perform in a recursive manner controlling its own data acquisition and processing modes.

The second architecture is a combined AI system that uses neural networks and two-dimensional filters to do first line object detection and locations of objects in an image input and then uses LISP type AI relationships to do object manipulation and image evaluation(figure 2). The advantage of this architecture is that it can be developed one detection algorithm and one relationship at a time. Neural networks and self-organizing nets have a unique ability to recognize patterns in two dimensional data (Carpenter and Grossberg 1991). Therefore, in this architecture the nets do the low level detection and pattern recognition and the other AI systems make decisions about what the patterns are based on relationships.

The third architecture is based on human perception theory and environmental theory. Off-line before the system is used, an environment is created in software to model the environment within which the system will operate. This model consists of a data base for the parameters of the environment and a set of rules and exceptions for the environment(see figure 3). Given the environment or environments, the online system works to detect the environment and then does its detection and image evaluation based on the expectations of that environment(Nevatia 1982).

The last architecture I will present is a blackboard. The blackboard architecture is an expert system architecture which uses several Knowledge Sources(KS) to develop information about the problem and then to manage this information in order to solve a complex inter-related problem(see figure 4 ). These systems are 
BLACK BOARD

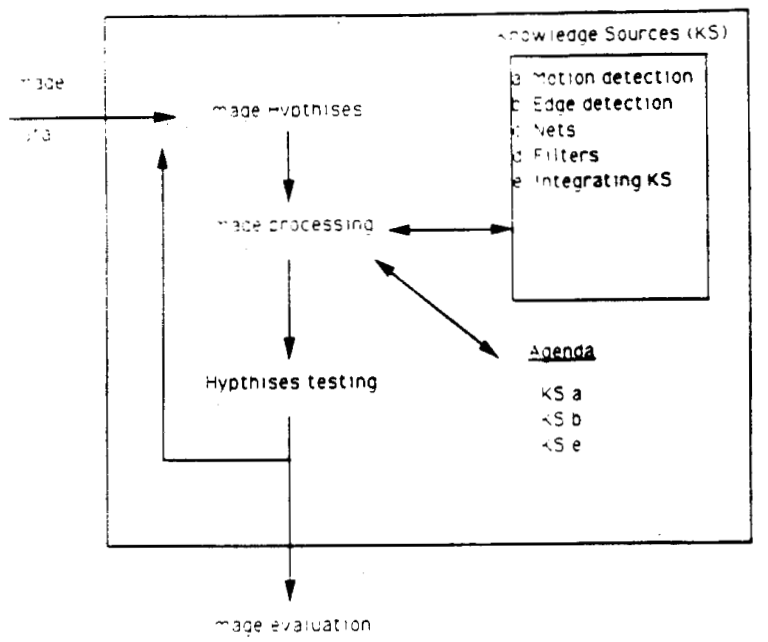

Figure 3:

now in use to solve many complex problems including some image processing work. The addition of neural networks and other more complex image technologies will add to their power to do computer vision tasks.

\section{Tools}

There are currently three major areas of development in computer vision algorithms. The first is low level computer detection algorithms. Some of these include line, shape, and object detection. The second area is the development of geometries from smaller less complex components. The last major area is the development of computer vision systems based strictly on modeling the human visual system. New computer vision tools will be developed and enhanced in this project. There are currently available a large number of statistical and frequency domain based image processing algorithms. Therefore, these traditional image processing algorithms will be used. The development of new tools will concentrate on neural nets and object identification tools. One of the new tools under development is eigan vector parameterization of classes of images. This technique decomposes parts of an image into areas of similarity with common objects and then identifies the object based on the amount

\section{ENVIRONMENTAL ARCHITECTURE-}

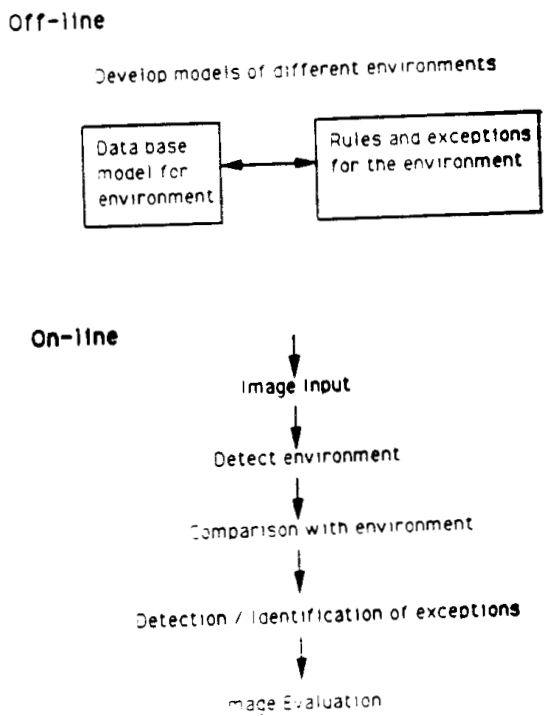

Figure 4:

of similarity to a known object. This method his considerable advantages in data compression. Thr.." is evidence that this is how some aspects of hum.i.it recognition is done. Also, I will continue my devil. opment of new neural nets to aid in computer visiun. Neural nets have proven useful in locating object. image data. The use of neural net in image procens. ing and computer vision is not only very powerful, but also allows the flexibility to develop systems based on given requirements of a project and still model the human visual system. I believe the development if these tools will allow us to develop more intelligent computer vision systems and understand more of the of the human visual system.

\section{Methods}

There will be several methods used to integrate and implement the systems used to "give" the compuin vision system the ability to make intelligent decision and gain awareness of the environment. The mode ing of the human vision system is based on neurop.: chology, the study of nerves in the brain. In of to model the intellectual functions of the human $v i$ sual system to improve performance and robustne... of computer systems, I will use some of the melt. 
ods of perception theory and enviormental phycology. Perception theory describes how and why humans direct their attention in order to obtain information and understand their environnment. I will use the principles of perception theory to direct the design of the AI systems. To the end of using the various tools and technologies available within the computer vision field to improve overall systems capabilities, I propose three methods. The first method is to develop new systems based on particular task demands that challenge the state of the art, in less constrained environments. Examples of these specific tasks could be robots that would function independently in a factory environment, or a general target recognizer for military use on the battle field. When developing systems for these difficult real world problems, we can quickly determine the flexibility and robustness of the systems we are developing. The second method is to study the humans vision systems in its relation to computer systems and the ability of human to perform many task an many environments. This study will help use to explore different combinations of systems for malty-purpose uses. The last method for improving the development of systems is to study the results of the first two studies in order ro find a aingle architecture which is generalizable.

\section{Conclusions}

The abilities and limitations of computer vision systems have considerable affects on the usability of many current and future computer systems and their operator interfaces. In this article I have reviewed and proposed a number of architectures of continued development of intelligent computer vision systems. I feel strongly that the use of combined artificial intelligence systems will provid the necessary capabilities for computer vision systems to begin operating in non-constrained environments.

\section{references}

1. Nevatia, Ramakant, Machine Perception, PrenticeHall, Inc. Englewood Ciffs, N.J. 1982

2. Carpenter, Gail A., Grossberg Steven, Pattern Recognition by Self Organizing Neural Networks, The MIT Press, Cambridge, Massachusetts, 1991 